\title{
Rendimiento académico de alumnos que asisten a escuelas para migrantes en México
}

\author{
Academic Achievement of Students who \\ Attend Schools for Migrants in Mexico
}

\author{
Carlos Rafael Rodríguez Solera \\ Universidad Iberoamericana Ciudad de México, México \\ carlosr.rodriguez@ibero.mx
}

\begin{abstract}
Juana María Islas Dossetti
Instituto Nacional para la Evaluación de la Educación (INEE), MÉxico jislas@inee.edu.mx

Patricia Patiño Martínez

Universidad Iberoamericana Ciudad de México, México

patricia.patino@ibero.mx
\end{abstract}

\section{RESUMEN}

Niñas y niños migrantes constituyen el grupo social con mayores problemas de exclusión educativa en México. Aunque ha aumentado la cantidad de migrantes que asisten a la escuela, la cobertura sigue siendo baja y la educación que reciben con muchas carencias. Es posible que los pocos que tienen acceso al sistema educativo, a su vez aprendan poco. No obstante, las revisiones de distintos documentos oficiales y estudios más actuales, revelan que no existen investigaciones sobre el logro académico de alumnos migrantes que permitan saber si las difíiciles condiciones en que estudian se traducen en bajos niveles de aprendizaje. Este estudio busca llenar este vacío mediante el análisis de la información disponible en bases de datos donde se identificó a las escuelas mexicanas que atienden a la población migrante, para obtener luego los datos relativos a los niveles de logro académico de sus alumnos. Se concluye que estos estudiantes presentan niveles de logro académico significativamente más bajos que los que asisten a otro tipo de escuelas; por esto, aun aquellos que permanecen en el sistema educativo hasta concluir la educación básica, no llegan a ejercer su derecho a recibir educación de calidad.

Palabras clave: niños, educación básica, migración, logro educativo, equidad educativa

\begin{abstract}
Migrant children constitute the social group with the greatest problems of educational exclusion in Mexico. Although the number of migrants attending school has increased, coverage remains low and the education they receive has many shortcomings. Hence, it is possible that the few migrants who have access to the educational system, in turn, learn little. However, there is no research on the academic achievement of migrant students that allow to know if the difficult conditions in which they study translate into low learning levels. This study seeks to fill this gap by analyzing the information available in databases of Mexican educational institutions. In these, the schools that serve the migrant population were identified, in order to obtain data on their students levels of academic achievement. The information allows us to conclude that these students present levels of academic achievement significantly lower than those who attend other types of schools, so even those who remain in the educational system until the completion of basic education, do not get to exercise, their right to receive quality education.
\end{abstract}

Key words: children, basic education, migration, educational achievement, educational equity 


\section{INTRODUCCIÓN}

Las niñas, niños y jóvenes (NNA) de familias de jornaleros agrícolas migrantes son los mexicanos que sufren mayores problemas de exclusión educativa en todo el país (Rodríguez, Juárez, Valdivieso y Mercado, 2014).

Como lo plantean varias investigaciones e informes sobre el tema (Rodríguez, 2006; Loyo, 2008; Sedesol, 2011; Chehaibar, Alcántara, Athié, Canales, Díaz-Barriga y Ducoing, 2013; Rodríguez et al., 2014; Schmelkes, Ramírez y Nesis, 2014; Rojas 2017a, 2017b; INEE, 2016a) los principales problemas que han enfrentado los migrantes en materia educativa ${ }^{1}$ son la falta de acceso a los servicios educativos y las dificultades para permanecer en la escuela.

Aunque la baja cobertura continúa siendo el principal problema que enfrenta la población migrante, existen otros de equidad educativa que afectan a dicha población y que escasamente se han abordado en las investigaciones sobre el tema. En particular, se desconocen cuáles son los niveles de aprendizaje que alcanzan los migrantes que logran acceder y permanecer en el sistema educativo hasta concluir la educación básica. Es importante aclarar que sí existen diversos estudios y publicaciones sobre esta población. ${ }^{2}$ Sin embargo, en ninguno de dichos documentos, se aborda el logro educativo de estos NNA.

El objetivo de la presente investigación fue conocer los niveles de logro que obtienen, en pruebas estandarizadas, las NNA migrantes que logran concluir los niveles de primaria y secundaria. Con

\footnotetext{
${ }^{1}$ Consideramos importante esta aclaración, debido a que, al problema educativo, se le suma la importante heterogeneidad y vulnerabilidad social y económica de dicha población; cuestiones que ya han sido documentadas en otras investigaciones (Rodríguez, 2009; Sedesol, 2011; Rojas, 2012; Miranda, 2015; INEE, 2016b, entre otras.)

${ }^{2}$ Destacan, por ejemplo, los realizados por el Fondo de Naciones Unidas para la Infancia (UNICEF, 2017), sobre niños, niñas y adolescentes (NNA) que viven en comunidades indígenas y el riesgo que enfrentan por no ir a la escuela o tener un bajo aprovechamiento académico; asimismo, los estudios sobre la protección especial que deben tener los NNA migrantes que transitan por México, entre otros. Por su parte, el Instituto Nacional para la Evaluación de la Educación (INEE) emitió, en 2016, un documento que incorpora un conjunto de recomendaciones para la atención educativa de esta población; en él se describe, en lo general, el estado de la situación educativa de esta población y se hace un importante balance sobre la política eductiva dirigida a ellos. Asimismo, varios investigadores han estudiado a esta población desde hace varios años, entre los que destacan Rodríguez, 2006; Loyo, 2008; Sedesol, 2011; Chehaibar et al., 2013; Rodríguez et al., 2014; Schmelkes et al., 2014; Rojas 2017a, 2017b, entre otros.
} 
esto se busca contribuir a llenar el vacío de conocimiento que existe sobre ese tema en las investigaciones sobre la educación a migrantes.

Para ello se procedió a analizar la información disponible en bases de datos de instituciones educativas mexicanas, con el propósito de identificar escuelas que atienden a la población migrante, cantidad de alumnos que asisten a dichos centros escolares y niveles de aprendizaje que obtienen en la prueba del Plan Nacional para la Evaluación de los Aprendizajes (Planea), en su modalidad de Evaluación de Logro referida a Centros Escolares (ELCE).

Los datos sobre la población estudiantil migrante están dispersos en varias fuentes de información, tales como el Sistema Nacional de Control Escolar de Población Migrante (Sinacem), el Sistema de Información y Gestión Educativa (Siged), la Dirección General de Educación Indígena (DGEI), y el Planea, entre otros, por lo que fue necesario conciliar, primero, las distintas bases de datos, a fin de identificar escuelas y alumnos migrantes dentro del universo total de centros escolares y de la matrícula reportada en distintas fuentes; a partir de ello, se reconstruye una numeralia, lo más fidedigna posible, sobre la población estudiantil migrante en México.

De acuerdo con la información analizada, en apariencia se han producido avances en el ejercicio del derecho a la educación por parte de niñas y niños migrantes, debido al incremento de la matrícula que, según los datos oficiales, se ha producido en los últimos cinco años. Sin embargo, no basta que los alumnos asistan a una escuela para que ejerzan de manera efectiva este derecho. También es necesario que, cuando acudan a los centros escolares, aprendan las competencias y conocimientos básicos que les permitirán, cuando sean adultos, desempeñar con éxito los roles económicos y sociales que demanda una sociedad moderna y democrática.

Los bajos resultados que los migrantes obtienen en las pruebas Planea muestran que, si bien ha aumentado su asistencia escolar, los aprendizajes que adquieren a su paso por la escuela son mínimos, lo que, sumado a los problemas de falta de acceso y a la poca permanencia que tienen en el sistema escolar, los perfila como el grupo social de México que padece las peores condiciones de exclusión educativa. Lo pocos que acceden a la educación formal salen tempranamente de ésta, antes de concluir la educación básica y, 
la ínfima cantidad de migrantes que logra terminar la secundaria, presentan bajísimos niveles de aprovechamiento escolar, por lo cual todavía estamos lejos de garantizarles su derecho a una educación de calidad con equidad.

\section{ENFOQUE CONCEPTUAL}

Existen diversos enfoques analíticos que pueden aplicarse al estudio de la equidad en educación. Si bien el presente estudio se sustenta en la propuesta de Farrell (2007), se considera necesario hacer algunas consideraciones teóricas que permitan recuperar y sistematizar distintas respuestas que se han dado en las ciencias sociales al problema de qué es una educación equitativa. En particular, se describen brevemente cuatro enfoques analíticos para abordar el tema de la equidad: el enfoque sobre el origen de la desigualdad, el de la igualdad fundamental, la teoría de la justicia de Rawls y la teoría de la independencia de esferas de Walzer.

a. Enfoque sobre el origen de la desigualdad: de acuerdo con este enfoque, una situación de equidad educativa sería aquélla en la que se hayan eliminado todas las barreras sociales, económicas y culturales que impiden a los alumnos el despliegue de sus capacidades potenciales. Este enfoque se remonta a la antigua discusión propuesta por Rousseau (1997), de distinguir entre las desigualdades justas, inevitables por ser de origen natural y las desigualdades injustas, evitables por ser producto de las instituciones sociales.

b. La teoría de la justicia de Rawls: en esta teoría, se enfatiza la necesidad de alcanzar la igualdad de oportunidades sobre un principio del maximin, es decir, beneficiando a los sectores menos favorecidos de la sociedad (Rawls, 2003). Así, en el caso de la educación, lo que se debe promover es la "igualdad equitativa de oportunidades", la sociedad debe establecer políticas que permitan iguales oportunidades de educación para todos, con independencia del ingreso de las familias de los alumnos.

c. La independencia de esferas de Walzer: sostiene que una educación equitativa es aquélla donde existe una igualdad de oportunidades, que permite desligar el desempeño escolar de la adscripción social de las personas. Michael Walzer (1983) sostiene que en una 
sociedad justa se debe impedir que las desigualdades que existen en una esfera social "contaminen" a otras esferas. Por ello, las desigualdades educativas deben ser independientes de las desigualdades observadas en otros ámbitos como el económico o el relativo al origen cultural.

d. Enfoque de la igualdad fundamental: este enfoque plantea que no se puede aspirar a una sociedad por completo igualitaria en todos los aspectos. Sin embargo, lo que sí se puede buscar es una sociedad equitativa, en la cual si bien van a seguir existiendo desigualdades, se promueve la igualdad en un aspecto que se considera fundamental para construir una sociedad justa (Rodríguez, 2008). Existen varias propuestas que aparecen en la literatura sobre el tema, en las que se intentan establecer criterios para identificar una igualdad fundamental en educación.

El Grupo Europeo de Investigación sobre Equidad del Sistema Educativo (EGREES por sus siglas en inglés), a partir de una propuesta formulada por Aletta Grisay, identifica al menos cinco principios para establecer qué se entiende por igualdad fundamental en educación: igualdad de acceso, igualdad de trato, igualdad de éxito o de logro, igualdad en el desarrollo de capacidades. Los criterios de equidad educativa que se emplean en cada país varían, según el énfasis que ponen en alguno de estos aspectos. Por ejemplo, si en una sociedad se busca la igualdad de trato, no habrá mucha preocupación por disminuir la desigualdad de logro, una consecuencia inevitable cuando se trata igual a estudiantes con distintas capacidades e intereses. Por el contrario, si lo que se valora es la igualdad de logro, se debe tolerar o incluso fomentar la desigualdad de trato, como lo que ocurre cuando se atienden las necesidades educativas de los grupos más vulnerables, asignando más o mejores recursos a su educación.

López (2005), a partir de una propuesta formulada por Demeuse, identifica, a su vez, cuatro formas en las que puede concebirse la igualdad fundamental: igualdad en el acceso, igualdad en las condiciones o medios de aprendizaje, igualdad en los logros o resultados e igualdad en la realización social de los logros educativos.

Bolívar (2005), basándose en planteamientos de Meuret y de Crahay, advierte la existencia de cuatro tipos de igualdad en educación: igualdad de oportunidades, igualdad de enseńanza, igualdad 
de conocimiento y éxito escolar e igualdad de resultados individuales y sociales.

Por su parte, Farrell (2007), entre otros autores, plantea la idea de que se deben promover de manera simultánea varias formas de igualdad. Por ello, el estudio se sustenta en la propuesta teórica de ese autor, según la cual, para que exista una condición de equidad educativa, todos los niños y las nińas deben tener igualdad de acceso al sistema educativo con independencia de su origen social o cultural o de la situación económica de su familia (equality of access). Por otra parte, debe existir igualdad de supervivencia escolar (equality of survival). Esto es, todos los alumnos que entran al sistema escolar deben mantenerse en él hasta concluir al menos la educación que se considera como obligatoria.

Un aspecto de fundamental importancia para la presente investigación es que, para que exista equidad educativa, debe alcanzarse también la igualdad en el logro (equality of output). Las niñas y nińos provenientes de diferentes agrupaciones sociales deben tener las mismas probabilidades de aprender las mismas cosas, a los mismos niveles, en un punto definido del sistema educativo.

Esta investigación intenta identificar, a partir de la información disponible en bases de datos publicadas por instituciones educativas, hasta qué punto los migrantes ejercen estos tres tipos de igualdad educativa o si, por el contrario, sufren diversas formas de exclusión que los perfilaría como uno de los grupos con mayores carencias, cuya superación requiere de una atención prioritaria mediante una política pública que busque la promoción de la equidad educativa.

\section{METODOLOGÍA}

La investigación busca solventar la falta de análisis sistemáticos sobre el logro educativo de nińas y niños migrantes. Un estudio en el que se analizan los resultados de investigaciones sobre equidad educativa, incluidas las enfocadas en las condiciones de niñas y niños migrantes, encontró que no existían investigaciones sobre su desempeño (Rodríguez et al., 2014).

Dicho resultado concuerda con lo expuesto en documentos oficiales sobre educación a migrantes. De acuerdo con las "Directrices 
para mejorar la atención educativa de niñas, niños y adolescentes de familias de jornaleros agrícolas migrantes", publicadas por el Instituto Nacional para la Evaluación de la Educación (INEE, 2016a), se cuenta con muy poca información para conocer, entre otros, los niveles de logro de aprendizaje que alcanzan los estudiantes en situación de migrantes.

Prácticamente no existe información sobre los materiales y contenidos educativos, la infraestructura y la gestión de los espacios en donde se imparten las clases en los campamentos o zonas de origen, los niveles de logro de aprendizajes de los alumnos en contexto migrante, así como la formación y perfiles de los docentes (INEE, 2016a, p. 61, el destacado es nuestro).

Dicha situación se ha originado por la dificultad que los investigadores han tenido para identificar, en los datos disponibles sobre el logro escolar, cuáles corresponden a los alumnos migrantes. Este problema metodológico fue señalado por el INEE, cuando sostiene que ha sido difícil conocer los niveles de logro de este tipo de alumnos debido a "la falta de mecanismos para identificar entre las niñas y los niños que participan en las evaluaciones, a quienes son parte de una familia de jornaleros agrícolas migrantes" (INEE, 2016a, p.6 2).

Es por ello que, en efecto, el principal problema metodológico del presente estudio fue identificar a los migrantes en las bases de datos existentes y, una vez hecho esto, observar los niveles de logro que obtuvieron en la prueba Planea ELCE.

Para aislar los datos de los migrantes, se procedió primero a identificar a las escuelas que reportan información al Sinacem. Una alternativa era considerar como migrantes a todos los alumnos cuyas escuelas aparecían en dicha base de datos. Sin embargo, no todas las escuelas que envían información al Sinacem se especializan en la educación a migrantes, por lo cual sería erróneo identificar de este modo a la población objetivo.

Otra alternativa es rastrear a las escuelas para migrantes de acuerdo con la clave que se usa para identificarlas. En México, cada centro escolar está registrado en el Sistema Educativo Nacional (SEN) con una Clave de Centro de Trabajo (CCT), la cual está conformada por cinco campos: 
1. Entidad federativa (dos caracteres): identifica a la entidad donde está ubicado el centro de trabajo; así, se numeran los estados en orden alfabético del 01 al 32.

2. Clasificador (un carácter): define al centro de trabajo (CT) de acuerdo con la naturaleza del financiamiento del servicio que presta. Éste puede ser Federal (D), Estatal (E) y Conafe (K).

3. Identificador (dos caracteres): identifica los tipos, niveles y servicios. El identificador ZC, se refiere a la primaria regular de migrantes (DOF, 2013), mientras que el ZS pertenece a secundarias para migrantes (SEP, 2017).

4. Número progresivo (cuatro caracteres): sirve para enumerar los centros de trabajo por cada entidad.

5. Elemento verificador (un carácter): se asigna computacionalmente en el momento de dar de alta un CT; su función es validar el correcto registro de la clave en todos los procesos en que ésta sea utilizada (Unidad de Planeación y Evaluación de Políticas Educativas, 2010).

A partir de estas normas fue posible identificar a las escuelas primarias para migrantes como aquellas cuyo CCT contenía el clasificador "D" y el identificador "ZC" y a las escuelas secundarias para migrantes, siendo éstas las que tuvieran el clasificador " $D$ " y el identificador "ZS".

Esta forma de identificación de las escuelas es usada además por el Siged en la definición de las Estructuras Ocupacionales al interior de la Secretaría de Educación Pública (SEP).

$\mathrm{Al}$ emplear el criterio de filtrar centros escolares cuyos CCT contuvieran las cadenas DZC y DZS se pudo identificar a las escuelas especializadas en la educación a migrantes. Como puede verse en la figura 1, la situación es compleja, porque las escuelas para migrantes pueden o no estar en Sinacem y pueden o no participar en las pruebas Planea ELCE.

Para los efectos de la presente investigación se definió como parte del objeto de estudio a todas aquellas escuelas para migrantes (DZC y DZS) que hubieran participado en Planea ELCE, ${ }^{3}$ con inde-

\footnotetext{
${ }^{3}$ Se utilizó esta modalidad de la prueba debido a que es la fuente más actual que aporta información por CCT. Si bien existe también la modalidad de Evaluación referida al Sistema Educativo Nacional (Planea ELSEN), ésta no se utilizó, debido a que no aporta información por CCT.
} 
pendencia de que estuvieran o no en Sinacem. Como se aprecia en el cuadro 1, en 2015 se identificó un total de 87 escuelas primarias y 19 escuelas secundarias.

Una vez identificadas las escuelas para migrantes, se consideró como "alumno migrante" a todos aquellos que estudian en una escuela de ese tipo, lo que permitió definir a la población cuyos niveles de logro académico se iban a observar. ${ }^{4}$

Cuadro 1. Número de escuelas en las combinaciones encontradas en las bases de datos de centros escolares que atendían a migrantes en 2015

\begin{tabular}{|c|c|c|c|}
\hline Situación & Descripción & Primarias & Secundarias \\
\hline A & Total de escuelas que aplicaron Planea. & $73769^{i}$ & $31635^{i}$ \\
\hline B & Total de escuelas reportadas en Sinacem. & $464^{i i}$ & $135^{\mathrm{ii}}$ \\
\hline c & $\begin{array}{l}\text { Aplicaron Planea y están en Sinacem, pero NO } \\
\text { son escuelas para migrantes. }\end{array}$ & $115^{\mathrm{iii}}$ & $83^{\mathrm{iii}}$ \\
\hline$d$ & $\begin{array}{l}\text { Son escuelas para migrantes, están en } \\
\text { Sinacem y aplicaron Planea. }\end{array}$ & $80^{\mathrm{iii}}$ & $15^{\mathrm{iii}}$ \\
\hline e & $\begin{array}{l}\text { Son escuelas para migrantes, están en } \\
\text { Sinacem, pero NO aplicaron Planea. }\end{array}$ & $210^{\mathrm{iii}}$ & $29^{\mathrm{iii}}$ \\
\hline$f$ & $\begin{array}{l}\text { Están en Sinacem, NO aplicaron Planea y NO } \\
\text { son escuelas para migrantes. }\end{array}$ & $59^{\mathrm{iii}}$ & $8^{\mathrm{iii}}$ \\
\hline \multirow[t]{3}{*}{ g } & $\begin{array}{l}\text { Son escuelas para migrantes, aplicaron Planea, } \\
\text { pero NO están en Sinacem. }\end{array}$ & $7^{\mathrm{iii}}$ & $4^{i i i}$ \\
\hline & $\begin{array}{l}\text { Total de escuelas para migrantes reportadas } \\
\text { en Sinacem. }\end{array}$ & $290^{\mathrm{ii}}$ & $44^{\mathrm{ii}}$ \\
\hline & $\begin{array}{l}\text { Total de escuelas para migrantes que } \\
\text { aplicaron Planea. }\end{array}$ & $87^{\mathrm{iii}}$ & $19^{\mathrm{iii}}$ \\
\hline
\end{tabular}

Fuentes: i. SEP (2018a); ii. Sistema Nacional de Control Escolar de Población Migrante. Infraestructura en Educación Migrante (2015); iii. Cálculos propios con base en SEP (2018a) y Sistema Nacional de Control Escolar de Población Migrante (2015).

\section{RESULTADOS}

\section{Características sociodemográficas de los migrantes}

Los jornaleros agrícolas migrantes constituyen una franja de la población cuyo sustento principal es el salario que reciben por trabajar

\footnotetext{
${ }^{4}$ Para el análisis de los datos se utilizó el software de análisis estadístico IBM Corp. (2016). IBM SPSS Statistics for Windows, Version 26.0. Armonk, Nueva York: IBM Corp.
} 
en el sector agrícola, fuera de su localidad de origen, y se emplean mayormente en las cosechas de café, tomate rojo, tomate verde, chile y durazno.

De acuerdo con la Encuesta Nacional de Jornaleros (Enjo), para $2009^{5}$ había 2099586 jornaleros agrícolas migrantes de los cuales cerca de 17.7 \% tenía entre 5 y 17 años de edad (INEE, 2016a). Su constante movilidad se explica, particularmente, por la pobreza estructural de sus comunidades de origen, pues 6 de cada 10 migrantes fueron expulsados de localidades de alta o muy alta marginación, donde el acceso a los servicios de salud, escuelas y sistemas de seguridad es limitado; a ello se suma la baja productividad de la tierra y escasas opciones de trabajo remunerado (Sedesol, 2011).

La vulnerabilidad es, sin duda, la carcaterística principal de esta población: cerca de $80 \%$, se encuentra en situación de pobreza multidimensional (Sedesol, 2011). Alrededor de 40\% de los jornaleros agrícolas migrantes pertenecen a una comunidad indígena y hay hablantes de 29 lenguas distintas (Sedesol, 2011). Destaca en este respecto que la población indígena, muestra mayores rezagos en su ingreso, una menor tasa de alfabetización y menos años promedio de escolaridad.

Por lo que hace a la población en edad escolar que los acompańa, se calcula que existen entre 279 y 326 mil niñas, niños y adolescentes de familias de jornaleros agrícolas migrantes en edad de cursar la educación básica, esto es, 1 de cada 100 niños en México pertenece a esta población (INEE, 2016a). Las condiciones de vulnerabilidad en las que viven estas niñas, niños, adolescentes y jóvenes, se configuran en un gran reto para garantizar su acceso, permanencia y logro educativo. A continuación, se muestra el estado de la situación al respecto.

\section{Numeralia de la educación a migrantes}

Un reto fundamental del presente estudio es identificar con precisión a las niñas y niños migrantes que son atendidos por el Sistema Educativo Nacional (SEN) y, dentro de ellos, a los que participaron

\footnotetext{
${ }^{5}$ La Encuesta Nacional de Jornaleros 2009 (Enjo) señala que 78 de cada cien jornaleros agrícolas se encuentran en situación de pobreza multidimensional (Sedesol, 2011b), lo cual refleja la grave situación de vulnerabilidad que enfrentan.
} 
en la prueba Planea ELCE, lo que permitiría conocer los niveles de logro académico de dicha población.

Los datos publicados por la Secretaría de Educación Pública identifican al total de alumnos en las escuelas clasificadas como "escuelas para migrantes" por nivel educativo (cuadro 2). ${ }^{6}$ La información está disponible para todas las entidades del país; sin embargo, estos datos deben tomarse con precaución, debido que esas cifras incluyen a alumnos migrantes en situaciones muy diversas. Tomemos por ejemplo los datos de 2015. De acuerdo con la Dirección General de Educación Indígena (DGEI/SEP, 2015), 25734 alumnos migrantes asistieron a primaria en 480 escuelas. Asimismo, 3112 estudiantes de educación secundaria fueron atendidos en 134 escuelas.

- Cuadro 2. México: Matrícula total de niñas y niños migrantes por nivel educativo, 2015-2018

\begin{tabular}{|r|c|c|c|}
\hline & \multicolumn{3}{|c|}{ Ciclo escolar } \\
\hline Nivel educativo & $\mathbf{2 0 1 5 - 2 0 1 6}$ & $\mathbf{2 0 1 6 - 2 0 1 7}$ & $\mathbf{2 0 1 7 - 2 0 1 8}$ \\
\hline Inicial & 2248 & 2197 & 3058 \\
\hline Preescolar & 8226 & 7885 & 9207 \\
\hline Primaria & 25734 & 27538 & 33248 \\
\hline Secundaria & 3112 & 2806 & 3417 \\
\hline Total & 39320 & 40426 & 48930 \\
\hline
\end{tabular}

Fuente: SEP, 2017 y 2018a.

No obstante, en las bases de datos consultadas sólo aparecen 290 escuelas para dicha población en primaria y 44 en secundaria (ver cuadro 1), las cuales se identifican por incluir en la Clave de Centro de Trabajo (CCT), las siglas DZC, exclusivas de las escuelas de educación primaria migrante y DZS, exclusivas de escuelas de educación secundaria migrante (SEP, 2019b).

Esta discrepancia ocurre porque la SEP incluye como "escuelas migrantes" a todos aquellos establecimientos que en el Sinacem informan atender a estudiantes en esa condición.

\footnotetext{
${ }^{6}$ En este ejercicio, se asumió como migrantes a todos los estudiantes matriculados en estas escuelas. No obstante, se asume que no se pudo corroborar, empíricamente, que el $100 \%$ lo fuera, en atención a las dificultades logísticas que ello significaría, y dado que no hay información oficial que permita corroborarlo.
} 
En otras palabras, en el Sinacem se inscriben escuelas regulares que cuentan entre sus estudiantes a algunos migrantes, pero que en modo alguno se especializan en atender a dicha población, por lo que su CCT no incorpora la clave DZC o DZS.

Para conocer los niveles de logro académico de los migrantes no se puede considerar como tales a los que estudian en todas las escuelas que reportan datos al Sinacem. Ello en atención a que, en dicho sistema aparecen tanto escuelas para migrantes como escuelas regulares y, en estas últimas, no es posible saber cuáles de los alumnos que aplicaron la prueba Planea ELCE son migrantes y cuáles no. Como se dijo ya en el apartado metodológico, el universo del presente estudio se define como "alumnos migrantes" exclusivamente a los que estudian en escuelas especializadas en la atención a migrantes, las cuales, en las bases de datos disponibles, se identifican con las claves DZC (Escuela de Educación Primaria Migrante) y DZS (Escuela de Educación Secundaria Migrante).

Una situación similar a la descrita ocurre en los otros periodos identificados en el cuadro 2, por lo cual siempre seguimos el mismo criterio de identificar como estudiantes migrantes sólo a los que asisten a escuelas que atienden de manera exclusiva a dicha población.

Las combinaciones encontradas en las bases de datos que aportan información sobre migrantes se ilustran en la figura 1 .

Figura 1: Combinaciones encontradas en bases de datos de escuelas que atienden a migrantes*

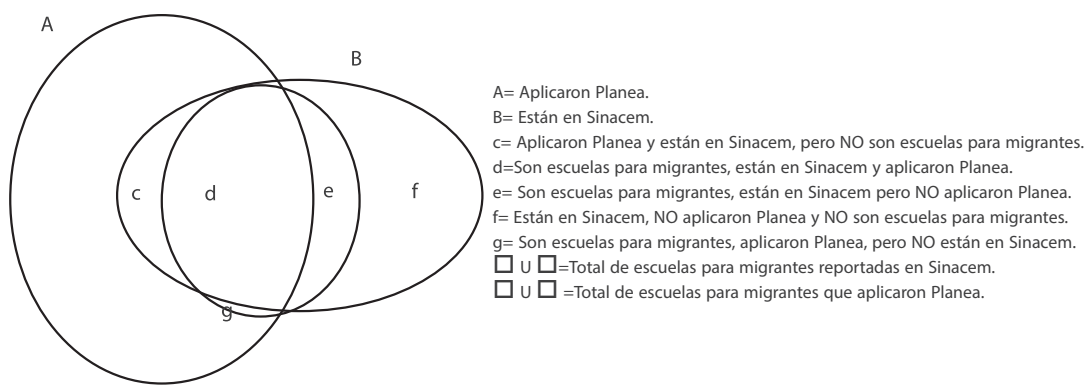

Fuente: elaboración propia. *Los tamaños de los círculos y elipses no son proporcionales a la cantidad de escuelas. 
De todas las situaciones descritas identificamos como universo de nuestro estudio a los centros escolares que están en las combinaciones "d" y "g", o sea, a las escuelas para migrantes que aplicaron Planea, con independencia de que estuvieran o no en la base de datos de Sinacem.

A partir de dichos criterios identificamos a las escuelas y a los estudiantes migrantes que aplicaron las pruebas Planea, como se especifica en la cuadro 3.

Cuadro 3. Total de centros escolares y alumnos en escuelas para migrantes que aplicaron la prueba Planea Escuelas, 2015-2018

\begin{tabular}{|c|c|c|c|c|c|c|c|c|}
\hline Nivel & \multicolumn{5}{|c|}{ Escuelas } & \multicolumn{5}{c|}{ Alumnos } \\
\hline Año & $\mathbf{2 0 1 5}$ & $\mathbf{2 0 1 6 ^ { * }}$ & $\mathbf{2 0 1 7}$ & $\mathbf{2 0 1 8}$ & $\mathbf{2 0 1 5}$ & $\mathbf{2 0 1 6}$ & $\mathbf{2 0 1 7}$ & $\mathbf{2 0 1 8}$ \\
\hline Primaria & 87 & 82 & $\begin{array}{c}\text { No hubo } \\
\text { aplicación }\end{array}$ & 73 & $\begin{array}{c}\text { LyC: } 383 \\
\text { Mat: } 394\end{array}$ & $\begin{array}{c}\text { LyC: } 343 \\
\text { Mat: } 341\end{array}$ & $\begin{array}{c}\text { No hubo } \\
\text { aplicación }\end{array}$ & $\begin{array}{c}\text { LyC: } 362 \\
\text { Mat: } 375\end{array}$ \\
\hline Secundaria & 19 & 19 & 22 & $\begin{array}{c}\text { No hubo } \\
\text { aplicación }\end{array}$ & $\begin{array}{c}\text { LyC: } 82 \\
\text { Mat: } 80\end{array}$ & $\begin{array}{c}\text { LyC: } 121 \\
\text { Mat: } 115\end{array}$ & $\begin{array}{c}\text { LyC: } 126 \\
\text { Mat: } 129\end{array}$ & $\begin{array}{c}\text { No hubo } \\
\text { aplicación }\end{array}$ \\
\hline
\end{tabular}

Fuentes: SEP, 2018a con datos para 2015, 2016, 2017 y 2018.

* Si bien se identificaron escuelas primarias y secundarias migrantes que aplicaron la prueba Planea en 2016, en el presente estudio, decidimos no hacer análisis ni inferencias sobre los niveles de logro de los estudiantes para este año. Ello en atención a que, con base en información y Documentos Internos, la aplicación de la prueba no cubrió con el protocolo establecido por el INEE, particularmente por el hecho de que no se contó con aplicadores externos, esto es, la prueba se aplicó a través de aplicadores internos.

Los análisis que presentaremos más adelante sobre el logro educativo de los migrantes se enfocarán en esta población.

\section{Acceso de los migrantes a la educación básica}

Como ya se dijo, los migrantes han tenido especiales dificultades para acceder al sistema escolar, lo cual ha motivado el impulso de programas gubernamentales, cuyo objetivo es ampliar la oferta educativa dirigida a los mismos, el principal de los cuales, por su alcance y cobertura, es El Programa de Educación Básica para Niños y Niñas de Familias Jornaleras Agrícolas Migrantes (Pronim) el cual se integró, en 2014, junto con otros seis que atienden a población en situación de vulnerabilidad de distinto tipo, al Programa para la Inclusión y la Equidad Educativa (PIEE). La educación de los migrantes se fortaleció, además, con la legislación que prohíbe el trabajo 
infantil y eleva la edad mínima de admisión al empleo de 14 a 15 años, a partir de la reforma constitucional del artículo 123, aprobada en 2014; asimismo, la política social se ha sumado a la política educativa y a la legislación laboral, por medio del otorgamiento de becas. En conjunto, estas políticas han impulsado un incremento de la asistencia escolar de los migrantes.

De acuerdo con los datos publicados por la Secretaría de Educación Pública (ver cuadro 1), en sólo tres años la atención a niñas y niños migrantes en educación inicial y básica se incrementó en 24.4\%. Dicho incremento obedeció a una tendencia histórica. En el ciclo escolar 1985-1986, el más antiguo para el que se dispone de información, sólo 3069 niñas y niños migrantes asistieron a la escuela (Rojas, 2011). Cuando comparamos los datos actuales de cobertura (48930 en 2018) con esa cifra tan baja, es claro que se ha producido un fuerte incremento en la cantidad de niñas y niños migrantes que estudian.

Se debe reconocer que las políticas gubernamentales han contribuido a que niñas y niños migrantes, antes excluidos, ahora tengan acceso a la educación básica. No obstante, el reto sigue siendo enorme. De acuerdo con el INEE (2016a, p. 18) la población total en edad de asistir a la escuela se estima entre 279000 y 326000 nińas y niños migrantes. Si se considera que 48930 estuvieron en algún centro escolar en 2018, eso implica que sólo entre 15.0 y $17.5 \%$ del total de esta población accedió a la educación básica, lo cual quiere decir que, en el caso de los migrantes, ocho de cada diez alumnos potenciales no ejercen de manera efectiva el derecho a la educación que les garantiza la legislación vigente.

Esta estimación es consistente con la que hace el INEE a partir de datos de la SEP:

Otro de los grupos en condiciones de vulnerabilidad es el de los niños pertenecientes a familias de jornaleros agrícolas migrantes, quienes provienen comúnmente de localidades pequeńas y con alto grado de marginación; muchos de ellos son indígenas y hablantes de alguna lengua indígena. Según estudios que retoman cifras de la SEP, se estima que sólo asiste entre 14 y $17 \%$ de los niños de familias de jornaleros migrantes en edad para cursar la EB (INEE, 2018a, p. 114). 
La exclusión del sistema educativo que sufren nińas y niños migrantes es la más alta observada en grupo alguno. Sólo para contar con parámetros de comparación, la tasa de asistencia escolar en el grupo de edad de 6 a 11 y de 12 a 14 años era, en 2015 , de $97.0 \%$ y $77.5 \%$ en hogares en pobreza extrema; $95.9 \%$ y $86.8 \%$ en los hablantes de lengua indígena; $96.6 \%$ y $88.8 \%$ en localidades de alto grado de marginación y $97.7 \%$ y $93.3 \%$ en la población en general (INEE, 2018a). Ello refleja, a todas luces, las enormes dificultades que enfrentan las niñas, niños y adolescentes migrantes para acceder a la educación.

\section{Permanencia de los migrantes en la educación básica}

No existen estudios que permitan cuantificar, en el caso de los migrantes, cuál es su permanencia en la educación básica, pero los datos disponibles permiten esbozar algunos rasgos generales. Un primer dato a destacar es la escasa cantidad de alumnos migrantes que logran cursar y concluir la educación secundaria. De acuerdo con los datos expuestos en el cuadro 2, en el caso de los migrantes se presenta una drástica disminución de la matrícula entre los niveles de primaria y secundaria.

En el ciclo 2016-2017 asistían a primaria 27538 alumnos migrantes, pero sólo 2806 niñas y niños migrantes asistían a la secundaria. En ese mismo ciclo escolar, en el nivel nacional asistieron 14137862 nińas y niños a primaria y 6710845 a secundaria (INEE, 2018b). Mientras en el país estaban matriculados dos alumnos en primaria por cada alumno de secundaria, en el caso de los migrantes esta proporción era de diez a uno. De acuerdo con estos datos, cuando se compara con lo que ocurre en la población en general, una proporción menor de niños migrantes se incorporan a la educación secundaria $y$, sin duda, los que logran incorporarse a dicho nivel educativo, tienen dificultades para concluirlo, dada su condición de migrantes.

Tanto en primaria como en secundaria los niños migrantes que acceden a la escuela tienen dificultades para continuar en ella hasta concluir sus estudios, lo cual se aprecia en la poca cantidad de escuelas para migrantes en las cuales se aplicó la prueba Planea en sexto 
de primaria en 2015, 2016 y 2018 y en tercer grado de secundaria para 2015, 2016 y 2017, así como la escasa cantidad de estudiantes que rindieron dicha prueba.

En el caso de primaria, en la prueba Planea 2015 participaron 87 centros escolares que representan sólo 29.2\% del total de escuelas primarias para migrantes que había en el país en ese año. Para el caso de secundaria, sólo se cubrió $39.6 \%$ del total.

Este dato da una idea de la poca cantidad de niñas y niños que llegan a cursar los grados terminales en escuelas primarias y secundarias para migrantes. Para una mayor comprensión de estas inferencias, se hace la siguiente aclaración. La prueba Planea ELCE es de carácter censal, y sólo se aplica en los grados terminales de la educación obligatoria $\left(6^{\circ}\right.$ de primaria y $3^{\circ}$ de secundaria para educación básica). La cobertura nacional de la prueba por escuela en 2015 fue de $88.7 \%$ para primaria y de $92.6 \%$ para secundaria (SEP e INEE, 2015). A nivel estado, en la mayoría de ellos, la prueba se aplicó sin contratiempos, alcanzando coberturas generales en educación básica por arriba de 96\%; salvo en Chiapas y Michoacán, donde la cobertura de aplicación fue menor $(37.2 \%$ y $18.3 \%$ de manera respectiva), debido al boicot de sindicatos que se opusieron a la prueba (SEP e INEE, 2015).

Cuando se analiza la misma información sólo para el caso de primarias y secundarias que atienden a población migrante, se observan coberturas bajas de aplicación (ver cuadro 4). Una hipótesis que nos permite explicar esta situación, alude al hecho de que, si bien en lo general, los estados que aparecen en el cuadro 4 reportan coberturas de aplicación elevadas (Baja California Sur, 99.1\%; Coahuila, 99.6\%; Colima, 100\%; Chihuahua, 96.8\%; Jalisco, 99.4\%; Sinaloa, 99.6\%; Sonora, 98.3\%; y Veracruz, 99.8\%), salvo en el caso de Coahuila, ésta disminuye cuando se analiza el caso de las escuelas para migrantes, debido a que, aun contando con ellas, la aplicación no se realizó debido a que no había, al momento de la prueba, alumnos migrantes inscritos en algún grado terminal. 
Cuadro 4. Total de escuelas primarias para migrantes con clave (DZC) y número de escuelas en las que se aplicó la prueba Planea en sexto grado, 2015

\begin{tabular}{|r|c|c|c|}
\hline \multicolumn{1}{|r|}{ Entidad } & Total de escuelas & Aplicaron Planea & $\%$ \\
\hline Baja California Sur & 23 & 12 & 52.2 \\
\hline Coahuila & 2 & 2 & 100.0 \\
\hline Colima & 7 & 5 & 71.4 \\
\hline Chihuahua & 17 & 10 & 50.9 \\
\hline Jalisco & 20 & 22 & 33.3 \\
\hline Sinaloa & 66 & 17 & 68.0 \\
\hline Sonora & 25 & 18 & 48.6 \\
\hline Veracruz & 37 & 87 & 44.2 \\
\hline Total en entidades donde NO se aplicó Planea & 100 & 0 & 0.0 \\
\hline Total en entidades donde se aplicó Planea & 197 & 87 & \\
\hline
\end{tabular}

Fuente: Cálculos propios con base en SEP, 2018a y Sistema Nacional de Control Escolar de Población Migrante. Infraestructura en Educación Migrante 2015. Documento interno.

En el caso de las escuelas de secundaria ocurre algo similar; sólo en 19 escuelas (39.6\%) se aplicó la prueba Planea 2015 (ver cuadro 5). Es probable que, en las otras 29 escuelas secundarias de ese tipo, no se aplicó la prueba porque no contaban con alumnos en tercero de secundaria. Las escuelas secundarias donde se aplicó Planea se localizan en los estados de Morelos (2), Sinaloa (6) y Sonora (11). Las 29 escuelas para migrantes donde no aplicó la prueba, bajo la hipótesis de que no contaban con grados terminales, son una muestra clara de la escasa proporción de alumnos que culminan la educación básica. 
Cuadro 5. Total de escuelas secundarias para migrantes con clave DZS y número de escuelas en las que se aplicó la prueba Planea en tercer grado, 2015

\begin{tabular}{|r|c|c|c|}
\hline \multicolumn{1}{|c|}{ Entidad } & Total & $\begin{array}{c}\text { Aplicaron } \\
\text { Planea }\end{array}$ & $\%$ \\
\hline Morelos & 2 & 2 & 100.0 \\
\hline Sinaloa & 17 & 6 & 35.3 \\
\hline Sonora & 12 & 11 & 91.7 \\
\hline Total en entidades donde se aplicó Planea & 31 & 19 & 61.3 \\
\hline Total en entidades donde NO se aplicó Planea & 29 & 0 & 0.0 \\
\hline TOTAL & 48 & 19 & 39.6 \\
\hline
\end{tabular}

Fuente: cálculos propios con base en: SEP, 2018a, con datos para 2015 y Sistema Nacional de Control Escolar de Población Migrante. Infraestructura en Educación Migrante 2015. Documento interno

Un importante dato a destacar es que, de las escuelas primarias y secundarias identificadas como "escuelas para migrantes" en las que se levantó Planea, y de las que se dispuso de información sobre el grado de marginación del municipio en que se ubican, entre $60 \%$ y $70 \%$ se encuentran en municipios de alta o muy alta marginación. Destaca en primaria el caso de Veracruz, en el que 13 de los 18 centros evaluados están en zonas de alta y muy alta marginación; en secundaria sobresale Sinaloa, en que $75 \%$ de las escuelas se ubican en municipios con la misma condición.

\section{Logro educativo de los migrantes en el nivel básico}

Como ocurre en otros sectores sociales que enfrentan problemas de exclusión educativa, al mejorar la cobertura, la atención se desplaza a los temas relacionados con la calidad, en especial con los niveles de logro académico de los alumnos.

Como lo hemos referido desde el principio de este documento, en el caso de los migrantes existe un gran vacío en el tema, debido, por una parte, a que la atención se ha centrado en los grandes problemas de cobertura que ha enfrentado esta población, lo cual hace que el bajo rendimiento escolar, de los pocos que asisten a la escuela, se vea como un problema menor. Por otra parte, los investigadores han enfrentado dificultades para identificar a los hijos de jornaleros 
agrícolas migrantes en el universo de alumnos evaluados en distintas pruebas estandarizadas del logro.

La presente investigación busca llenar este vacío, mediante el cotejo de los resultados que obtuvieron en la prueba Planea ELCE los estudiantes de las escuelas de educación primaria y secundaria migrantes. Este procedimiento permitió identificar los datos de rendimiento académico de los alumnos migrantes de sexto de primaria en 2015 y 2018, así como los resultados de alumnos de tercer grado de secundaria de 2015 y 2017.

La prueba Planea ELCE "agrupa los resultados obtenidos por los estudiantes en cuatro niveles de logro que informan acerca de los aprendizajes clave que deben ser adquiridos por los estudiantes y en qué medida se han apropiado de ellos ... estos niveles van del I al IV en orden progresivo, es decir, el nivel más bajo es el I y el más alto es el IV” (SEP, 2018a, pp. 4-5).

Los últimos resultados nacionales para primaria (2018) y secundaria (2017), señalan que, en educación primaria, 44.4\% de estudiantes evaluados tiene conocimientos insuficientes en Lenguaje y Comunicación, y $55.3 \%$ en Matemáticas; en secundaria, 28.8\% cuenta con resultados insuficientes en Lenguaje y Comunicación, y 59.5\% en Matemáticas.

Los resultados de aprendizaje por tipo de escuela son contrastantes (ver cuadros 6 y 7). De 2015 a 2018, los resultados de Planea de $6^{\circ}$ de primaria tanto en Lenguaje y Comunicación, como en Matemáticas, muestran que los estudiantes de escuelas migrantes presentan los niveles de logro educativo más bajos. La misma situación se observa en secundaria de 2015 a 2017.

Los datos sobre la proporción de alumnos migrantes de sexto grado de primaria con nivel insuficiente en la prueba Planea, tanto en Matemáticas como en Lenguaje y Comunicación, muestra que ocho de cada diez estudiantes concluyen la primaria sin dominar los niveles mínimos de aprendizaje que deben tener los alumnos en ese nivel educativo. 
Cuadro 6. Porcentaje de estudiantes con nivel insuficiente (Nivel I) por tipo de escuela en primaria, 2015 y 2018

\begin{tabular}{|r|r|r|r|r|r|r|r|}
\hline \multirow{2}{*}{$\begin{array}{r}\text { Grado y } \\
\text { año de la } \\
\text { evaluación }\end{array}$} & \multirow{2}{*}{ Asignaturas } & \multicolumn{6}{|c|}{ Tipo de escuela } \\
\cline { 3 - 8 } & Nacional & Comunitaria & $\begin{array}{r}\text { General } \\
\text { Pública }\end{array}$ & Indígena & Privada & Migrante \\
\hline $\begin{array}{r}6^{\circ} \text { de } \\
\text { primaria } \\
(2015)\end{array}$ & $\begin{array}{r}\text { Lenguaje y } \\
\text { Comunicación }\end{array}$ & 44.4 & 70.4 & 46.5 & 70.4 & 15.0 & 77.0 \\
\cline { 2 - 9 } & Matemáticas & 55.3 & 66.9 & 57.3 & 69.4 & 31.2 & 80.7 \\
\hline $6^{\circ}$ de & $\begin{array}{r}\text { Lenguaje y } \\
\text { primaria } \\
(2018)\end{array}$ & 45.2 & 68.3 & 47.6 & 70.2 & 16.4 & 74.0 \\
\cline { 2 - 9 } & Matemáticas & 55.1 & 73.5 & 57.4 & 72.8 & 29.9 & 79.7 \\
\hline
\end{tabular}

Fuente: elaboración propia con base en SEP, 2018a y 2018b.

Como se muestra en la gráfica 1 , esto revela que existe una enorme brecha en los niveles de aprendizaje entre los alumnos migrantes y los que asisten a otro tipo de centros escolares. En dicha gráfica se presentan solamente los resultados en Lenguaje y Comunicación de 2018, pero una situación similar se presenta en Matemáticas y, de acuerdo con los datos del cuadro 6, en 2015 se presentaron brechas similares entre los niveles de aprendizaje de los migrantes, en relación con los alumnos que son atendidos en otros tipos de servicio educativo.

Gráfica 1. Porcentaje de estudiantes con nivel insuficiente (Nivel I) en Lenguaje y Comunicación en 6to de primaria, por tipo de escuela, 2018

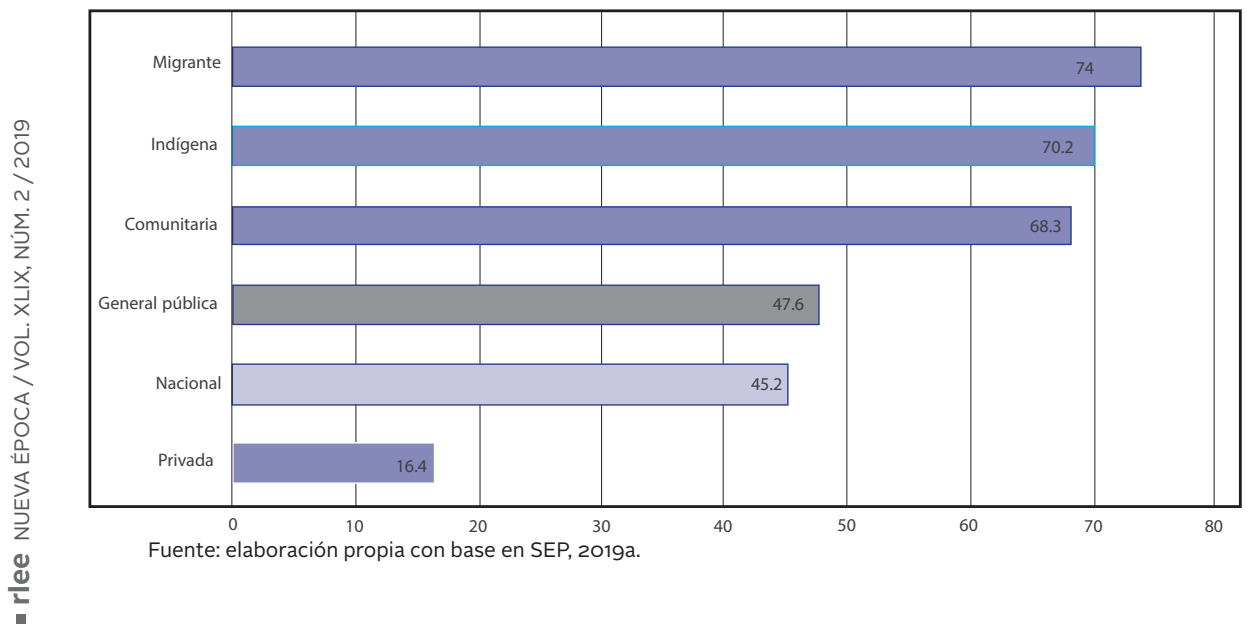


Estas brechas educativas son más preocupantes si se considera que, en el caso de los migrantes, la gran mayoría sólo llega a cursar el nivel de primaria, por lo cual, lo poco que aprendan en dicho nivel educativo será, para ellos, lo único con lo que pueden contar el resto de su vida. Su breve paso por el sistema escolar no se aprovecha para que adquieran las competencias que debe transmitir la educación básica.

En el caso de los pocos migrantes que logran terminar la educación secundaria, la situación es aún peor. Como se aprecia en el cuadro 3, menos de 130 alumnos, en todo el país, han participado en las pruebas Planea ELCE en escuelas secundarias para migrantes.

Cuadro 7. Porcentaje de estudiantes con nivel insuficiente (Nivel I) por tipo de escuela secundaria, 2015 y 2017

\begin{tabular}{|r|r|c|c|c|c|c|c|c|}
\hline \multirow{2}{*}{$\begin{array}{c}\text { Grado y } \\
\text { año de la } \\
\text { evaluación }\end{array}$} & \multirow{2}{*}{ Asignaturas } & \multicolumn{7}{|c|}{ Tipo de escuela } \\
\cline { 3 - 9 } & Nacional & Comunitaria & $\begin{array}{c}\text { General } \\
\text { pública }\end{array}$ & Privada & $\begin{array}{r}\text { Técnica } \\
\text { pública }\end{array}$ & Telesecundaria & Migrante \\
\hline $\begin{array}{r}3^{\circ} \mathrm{de} \\
\text { secundaria } \\
(2015)\end{array}$ & $\begin{array}{r}\text { Lenguaje y } \\
\text { Comunicación }\end{array}$ & 28.8 & 44.3 & 29.5 & 11.1 & 30.1 & 33.7 & 48.8 \\
\hline $\begin{array}{r}3^{\circ} \mathrm{de} \\
\text { secundaria } \\
(2017)\end{array}$ & $\begin{array}{r}\text { Lenguaje y } \\
\text { Comunicación }\end{array}$ & 34.8 & 58.8 & 34.2 & 12.5 & 34.9 & 45.0 & 75.4 \\
\hline
\end{tabular}

Fuente: elaboración propia con base en SEP, 2018a y 2018b, con datos para 2015 y 2017.

El solo hecho de concluir el tercer grado de secundaria, en las difíciles condiciones en que vive y trabaja esa población es, por sí mismo, admirable. Pero la pregunta es si el enorme esfuerzo que hacen las niñas y niños migrantes y sus familias se traduce en aprendizajes que permitan a esos adolescentes mejorar sus condiciones de vida, una vez que salgan de la escuela.

El aprovechamiento de las habilidades que transmite el sistema escolar para mejorar las condiciones de vida es algo que puede ser ajeno a la escuela. Sin embargo, lo que sí se puede observar es si el sistema educativo está cumpliendo con el deber de transmitir, al menos, los aprendizajes que ese mismo sistema ha definido como básicos o mínimos.

En el caso de los alumnos migrantes que logran concluir el tercer grado de secundaria, los datos que se exponen en el cuadro 7 ponen 
en evidencia que la mayoría egresa de la educación básica sin alcanzar los niveles mínimos de aprendizaje. En 2017, en esta situación estaban tres de cada cuatro alumnos, de acuerdo con la prueba de lenguaje y comunicación, y nueve de cada diez en la prueba de matemáticas.

Gráfica 2. Porcentaje de estudiantes con nivel insuficiente (Nivel I) en Matemáticas en zero de secundaria, por tipo de escuela, 2017

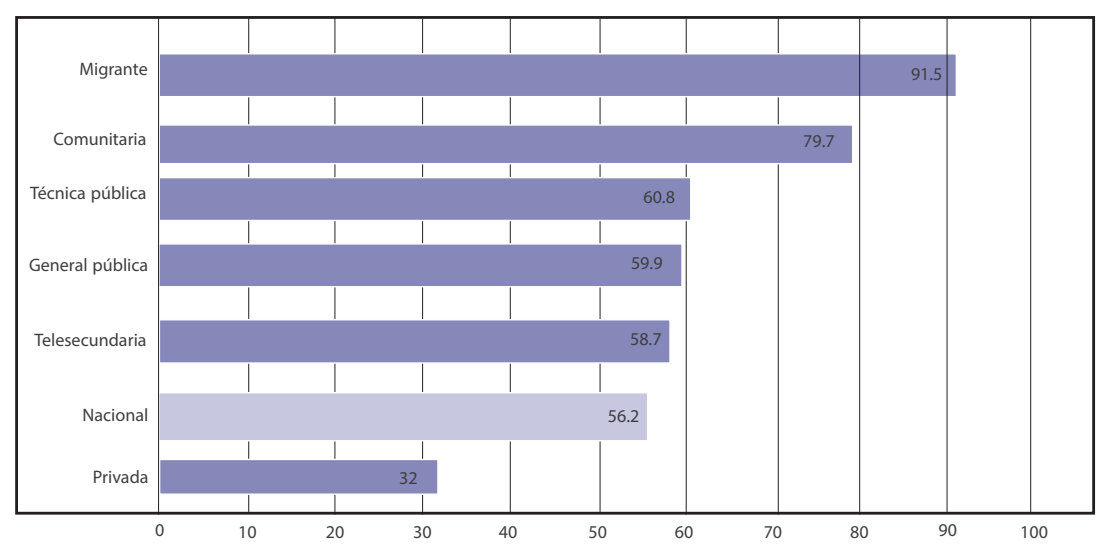

Fuente: elaboración propia con base en SEP, 2018a y 2018b.

De nuevo, las brechas entre los alumnos migrantes y los que asisten a otro tipo de escuelas se tornan dramáticas. En la gráfica 2 se exponen únicamente los resultados de la prueba de Matemáticas en 2017, pero una situación similar se observa en la otra materia evaluada (Lenguaje y Comunicación) y el otro año para el que se dispone de información (2015).

Esta situación también se refuerza con la poca mejora en los resultados de las y los estudiantes en las escuelas migrantes, en especial en el nivel secundaria. Si bien a nivel nacional se observa una tendencia incremental respecto a la proporción de estudiantes en los niveles de aprovechamiento más altos, en el caso de las escuelas migrantes esta tendencia no se presenta (ver gráficas 3 y 4).

Así, podemos afirmar que el Sistema Educativo Nacional sigue en deuda con las niñas, niños y adolescentes migrantes, no sólo porque no se han podido garantizar trayectorias de acceso y permanen- 
cia exitosas, sino también por el hecho de que, los pocos estudiantes que logran acceder y permanecer, no logran los aprendizajes esenciales para su desarrollo académico y personal.

Gráfica 3. Porcentaje de estudiantes evaluados en Lenguaje y Comunicación en secundaria que se ubicaron en los grupos de desempeño III y IV, 2015 y 2017.

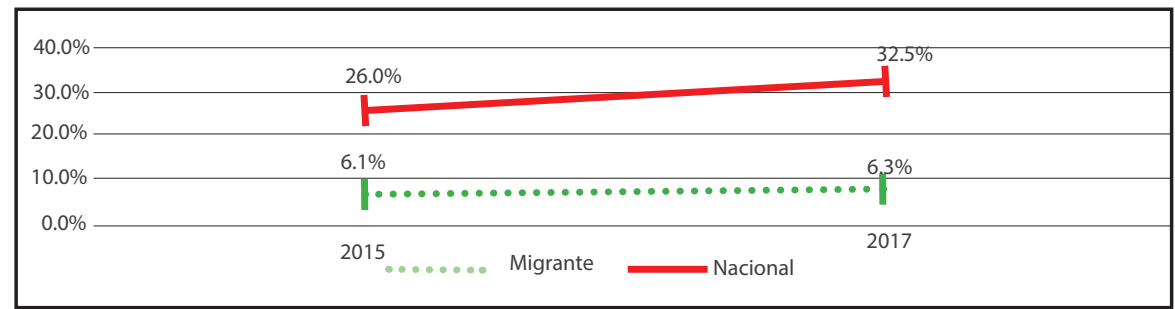

Fuente: elaboración propia con base en SEP, 2018a y 2018b.

Gráfica 4. Porcentaje de estudiantes evaluados en Matemáticas en secundaria que se ubicaron en los grupos de desempeño III y IV, 2015 y 2017.

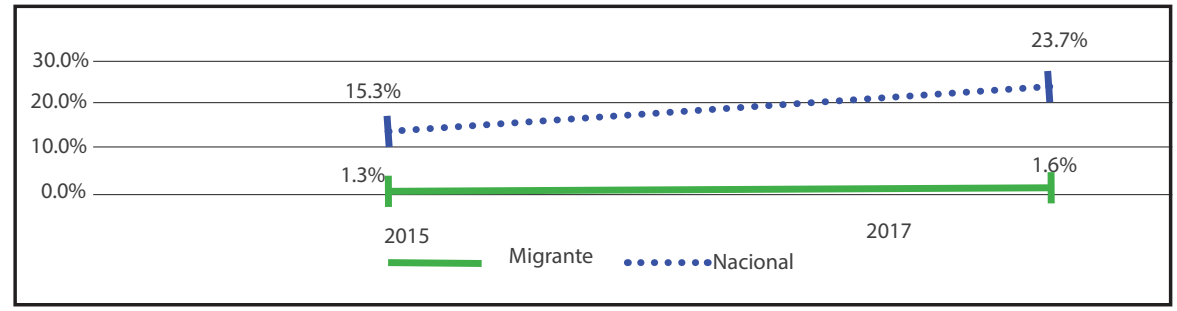

Fuente: elaboración propia con base en SEP, 2018a y 2018b.

\section{REFLEXIÓN FINAL}

Retomando la propuesta de Farrell (2007), mencionada al inicio del artículo, una situación de plena equidad educativa para la población migrante se daría cuando todos los niños y nińas tengan acceso a las escuelas, puedan permanecer en ellas hasta culminar sus estudios y cuando en su paso por la escuela adquieran los mismos conocimientos que alcanzan otros estudiantes que cursan la misma cantidad de grados. 
¿Cuánta educación deben recibir los niños y jóvenes? Al menos la educación definida como obligatoria en la legislación vigente. En este estudio sólo hemos mencionado el escaso acceso que la población migrante tiene a la educación básica, pero un análisis más detallado debería incluir también su acceso a la educación media superior, la cual también es ahora obligatoria.

A partir de los datos analizados en el presente estudio, la conclusión a la que se puede llegar es que niñas, niños y jóvenes migrantes experimentan una de las peores formas de exclusión educativa.

Pocos logran tener acceso a la educación formal (entre 1\% y $17 \%$ de la población potencial ${ }^{7}$ ) por lo que no existe la equality of access que plantea Farrell. De los que acceden al sistema educativo, muy pocos llegan a permanecer en él hasta completar al menos el tercer grado de secundaria, lo cual se aprecia en la poca cantidad de alumnos que aplican las pruebas Planea ELCE, exclusivas para alumnos en el último ańo de secundaria (ver cuadro 3), por lo que tampoco se cumple el principio de equality of survival de Farrell.

Por último, tal como lo muestran los datos de Planea ELCE tanto para primaria como para secundaria, los pocos niños y niñas que culminan la educación básica no llegan a aprender las mismas cosas que aprendieron otros alumnos, que alcanzaron los mismos niveles en el sistema educativo, por lo que tampoco se cumple con el principio de equality of output, propuesto también por Farrell.

En otras palabras, pocos migrantes acceden a la escuela, de los pocos que llegan a asistir, poquísimos son los que continúan hasta terminar el tercer grado de secundaria y, de éstos, la mayoría sale sin dominar los niveles mínimos de aprendizaje.

En el marco del ejercicio analítico realizado y, tomando también como referencia los estudios y evaluaciones que en los últimos años se han hecho a la política educativa orientada a la atención de esta población, podemos afirmar que ésta enfrenta todavía diversos cuellos de botella en su diseño, implementación y sobre todo, en sus resultados. Por lo que esperamos que este documento aporte al debate y llame a la reflexión y a la acción, a autoridades educativas,

\footnotetext{
${ }^{7}$ Cálculos propios según matrícula total de niñas y niños migrantes por nivel educativo en el periodo 2017-2018 (48930, SEP, 2018b) y estimación del número de niñas, niños y adolescentes migrantes agrícolas en edad de estudiar la educación básica (entre 279 y 326 mil, INEE, 2018a).
} 
legisladores y a organizaciones de la sociedad civil, para que, desde sus particulares trincheras, y en el marco de sus atribuciones legalmente definidas, desarrollen acciones suficientes y pertinentes para reducir las brechas de equidad que enfrentan los niños, niñas y adolescentes migrantes, quienes sin duda, padecen las consecuencias de la exclusión social y cultural, así como la vulnerabilidad económica en las que viven sus familias.

Finalmente, y si bien el objetivo de este artículo no fue la emisión de recomendaciones de política, consideramos importante señalar la urgencia de impulsar la inclusión y visibilización tanto de los NNA jornaleros agrícolas migrantes como de los servicios educativos que los atienden en las diferentes mediciones y estudios de logro educativo, condiciones de enseñanza y aprendizaje, y de política educativa que se realizan en el país. Ello será fundamental para contar con información suficiente y precisa, tanto de los aprendizajes que alcanza esta población, como de los servicios educativos que reciben, y que, sobre la base de dicha información, las autoridades educativas competentes tomen las mejores decisiones para garantizar a esta población su derecho a una educación de calidad.

\section{REFERENCIAS BIBLIOGRÁFICAS}

Bolívar, A. (2005). Equidad educativa y teorías de la justicia. Revista Iberoamericana sobre Calidad, Eficacia y Cambio en Educación, 3(2), 4269.

Chehaibar, L. M., Alcántara, A., Athié, M. J., Canales, A., Díaz-Barriga, A., y Ducoing, P. (2013). Diagnóstico de la educación. En UNAM (ed.), Plan Educativo Nacional (pp. 21-58). México: UNAM. Recuperado de http://www.planeducativonacional.unam.mx/PDF/completo.pdf

DGEI/SEP (2015). Principales cifras de educación indígena nacional. 2014-2015. Ciudad de México. Recuperado de https://dgei.basica. sep.gob.mx/files/prontuarios-estadisticos/pe_2014-2015.pdf

DOF (2013). Acuerdo por el que se emiten las Reglas de Operación del Programa de Desarrollo Humano Oportunidades, para el ejercicio fiscal 2013. México: Gobierno de México. Recuperado de http://dof. gob.mx/nota_detalle_popup.php?codigo=5289882 
Farrell, J. (2007). Equality of Education: Six Decades Seen from a New Millenium. En R. F. Arnove, C. A. Torres, \& F. Stephen. (Eds.), Comparative Education: The Dialectic of the Global and the Local (pp. 149-178). Rowman \& Littlefield Publishers.

INEE (2016a). Directrices para mejorar la atención educativa de niñas, niños $y$ adolescentes de familias de jornaleros agricolas migrantes. Ciudad de México: INEE.

INEE (2016b). La Educación Obligatoria en México. Informe 2016. Ciudad de México: INEE. Recuperado de https://publicaciones.inee.edu. $\mathrm{mx} /$ buscadorPub/P1/I/241/P1I241.pdf

INEE (2018a). La educación obligatoria en México. Informe 2018. Ciudad de México: INEE. Recuperado de https:/www.inee.edu.mx/portalweb/informe2018/04_informe/capitulo_020201.html

INEE (2018b). Principales cifras educación básica y media superior. Inicio del ciclo escolar 2016-2017. Ciudad de México: INEE.

López, N. (2005). Equidad educativa y desigualdad social. Desafíos de la educación en el nuevo escenario latinoamericano. Buenos Aires: Instituto Internacional de Planeamiento de la Educación, UNESCO.

Loyo, A. G. (2008). Evaluación Externa. Programa de Educación Preescolar y Primaria para Niñas y Niños de Familias Jornaleras Agrícolas Migrantes (PRONIM). México: UNAM.

Miranda, F. (2015). Preescolares Migrantes. Diagnóstico y propuestas de politica pública. México: Praxis.

Rawls, J. (2003). Teoría de la justicia. México: Fondo de Cultura Económica. Rodríguez, C. (2006). Informe final. Educación de migrantes en Hidalgo. México: Universidad Autónoma de Hidalgo.

Rodríguez, C. (2008). Equidad de la educación en México. Propuesta de un sistema de indicadores. Revista Perspectivas Sociales / Social Perspectives, $10(2), 55-79$.

Rodríguez, C. (2009). Evaluación externa del Programa de Educación Preescolar y Primaria para Niñas y Niños de Familias Jornaleras Agrícolas Migrantes (PRONIM) 2008. México: Universidad Autónoma del Estado de Hidalgo.

Rodríguez, C. R., Juárez, D., Valdivieso, A. G., y Mercado , F. (2014). Estudio sobre los principales resultados y recomendaciones de la investigación educativa en el eje de equidad. Ciudad de México: INEE.

Rousseau, J. J. (1997). Discurso sobre el origen y los fundamentos de la desigualdad entre los hombres. Valencia: Tilde. 
Rojas, T. (2011). Inequidades. La educación primaria de niños y niñas jornaleros agrícolas migrantes. México: UPN.

Rojas, T. (2012). Bienestar social de las familias agrícolas migrantes: acciones gubernamentales de las empresas agroexportadoras. Iberofórum. Revista de Ciencias Sociales de la Universidad Iberoamericana, 7(14), 3571. Recuperado de http://www.redalyc.org/pdf/2110/211026873002. pdf

Rojas, T. (2017a). Migración rural jornalera en México: la circularidad de la pobreza. Iberofórum. Revista de Ciencias Sociales de la Universidad Iberoamericana, 12(23), 1-35. Recuperado de http://www.redalyc.org/ pdf/2110/211053027001.pdf

Rojas, T. (2017b). Hijos de jornaleros migrantes, sin oportunidades para asistir a la escuela. La Jornada del Campo. Recuperado de http://www. jornada.unam.mx/2017/07/16/ cam-migrantes.html

Schmelkes, S., Ramírez, N., y Nesis, F. (2014). El trabajo infantil y el derecho a la educación en México. México: Fundación Telefónica México/ Paidós.

Sedesol (2011). Pobreza, migración y capacidades básicas en la población jornalera agrícola en México. Resultados de la encuesta nacional de jornaleros agrícolas 2009. México: Sedesol. Recuperado de http:// www.cipet.gob.mx/jornaleros/

SEP e INEE (2015). Plan Nacional para la Evaluación de los Aprendizajes, Planea en la Educación Básica 2015. Recuperado de http://planea. sep.gob.mx/content/general/docs/2015/difusion_resultados/2_Planea_Basica_2015_ELCE.pdf

SEP (2017). Censo de escuelas, maestros y alumnos de educación básica y especial. México. Recuperado de https://datos.gob.mx/busca/dataset/ censo-de-escuelas-maestros-y-alumnos-de-educacion-basica-y-especial SEP (2018a). Planea en Educación Básica. México: SEP. Recuperado de http://planea.sep.gob.mx/ba/

SEP (2018b). Información estadística nacional 2017-2018. México: SEP. Recuperado de https://dgei.basica.sep.gob.mx/files/infografias/2017-2018/nacional_2017_2018.pdf

UNICEF (2017). Educación y aprendizaje. La educación les da a niñas, niños y adolescentes las habilidades y conocimientos para alcanzar su máximo potencial y ejercer sus otros derechos. México: UNICEF. Recuperado de https://www.unicef.org/mexico/educaci\%C3\%B3n-yaprendizaje 
Unidad de Planeación y Evaluación de Políticas Educativas. (2010). Manual de Procedimientos para la operación del Catálogo de Centros de Trabajo. Guanajuato, México: Dirección General de Planeación y Programación. Secretaría de Educación Pública. Recuperado de http://www.seg.guanajuato.gob.mx/Ceducativa/SIIE/Normativa/Manual_operaci\%C3\%B3n_del_cat\%C3\%A1logo_de_centros_de_trabajo_2010.pdf

Walzer, M. (1993). Las esferas de la justicia: Una defensa al pluralismo y a la igualdad. México: Fondo de Cultura Económica.

\section{Agradecimientos}

Los autores agradecen a Eduardo Roberto Carlos Aguińaga Rincón por su apoyo en el procesamiento de datos. 